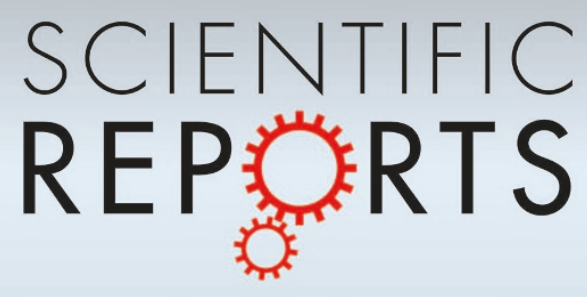

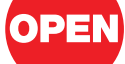

SUBJECT AREAS:

NANOWIRES

NANOPHOTONICS AND

PLASMONICS

ORGANIC-INORGANIC NANOSTRUCTURES

POLYMERS

Received

15 January 2013

Accepted

2 April 2013

Published

17 April 2013

Correspondence and requests for materials should be addressed to B.L. (stslbj@mail.sysu. edu.cn)

\section{Wavelength-converted wave-guiding in dye-doped polymer nanofibers}

\author{
Huaqing $\mathrm{Yu}^{1,2} \&$ Baojun Li ${ }^{1}$
}

\begin{abstract}
${ }^{1}$ State Key Laboratory of Optoelectronic Materials and Technologies, School of Physics and Engineering, Sun Yat-Sen University, Guangzhou 510275, China, ${ }^{2}$ School of Physics and Electronic-information Engineering, Hubei Engineering University, Xiaogan 432000, China.
\end{abstract}

Nanoscale wavelength-converted optical components are promising components for communication and optical information processing in integrated photonic system. In this work, we report a facile strategy for realizing continuously tunable wavelength-converted wave-guiding in dye-doped nanofibers. The nanofibers with diameters of 200-800 $\mathrm{nm}$ have an absorption coefficient of about $80 \mathrm{~cm}^{-1}$ and a self-absorption coefficient of about $30 \mathrm{~cm}^{-1}$, and exhibit relatively high PL efficiency and high photobleaching resistance under an optical pump. By launching the pump light into the nanofibers, the excited light in the nanofibers got self-absorption and reemitted at a longer wavelength, resulting in a gradual wavelength conversion during propagation. On the basis of this wavelength-converted wave-guiding, nanoscale wavelength-converted splitters were demonstrated by assembling the nanofibers into crossed structures. We believe that the dye-doped nanofibers would bring new exciting opportunities in developing new wavelength-converted optical components for nanophotonic device integration.

W ith the development of all-optical signal processing in compact photonic integrated system, nanophotonic devices is challenging, in which nanoscale wavelength-converted optical devices including wavelength converter ${ }^{1,2}$, wavelength router ${ }^{3}$, photonic beam splitter $^{4}$, wavelength multiplexer ${ }^{5,6}$, and wavelength-selective optical filter ${ }^{7,8}$, are key elements for their potential applications in optical information processing and communication. Therefore, several structures such as diffraction grating ${ }^{9}$, silicon-on-insulator waveguide $^{10}$, photonic crystal waveguide ${ }^{11}$, and dielectric-loaded plasmonic waveguide ${ }^{12}$ have been proposed to realize nanoscale wavelength-converted optical components. Recently, optical nanowires have received intense attentions due to their excellent flexibility, configurability, large evanescent fields and high nonlinearity. Therefore, several approaches including electron-beam lithography ${ }^{11}$, thermal evaporation ${ }^{13}$, and templating ${ }^{14}$ have been developed for producing nanowire-based wavelength-converted optical devices. These methods are, however, relatively complex (e.g., involve complicated equipments or require precise control over structure parameters such as lattice constants, composition, etc.), and costly for fabrication of the nanowires.

Polymer materials possess many attractive properties including biocompatibility, good processability, high flexibility, tunable properties, and low cost for integration ${ }^{15}$. A series of passive polymer components have been fabricated by Xing et al. using poly(trimethylene terephthalate) (PTT) nanofibers which were drawn from the PTT melt $^{16}$. More importantly, polymers can host a variety of functional dopants including quantum dots and fluorescent dyes that can be used to tailor their properties with greater versatility. Recently, lasing action and single wavelength conversion have been realized by incorporating fluorescent dyes into polymer nanofiber ${ }^{17,18}$. Polymer nanofibers have been drawn from rhodamine $\mathrm{B}(\mathrm{RhB})$ and polystyrene (PS) mixed chloroform droplet $^{17}$, but the self-absorption coefficient of the RhB-PS nanofiber is relatively high $\left(50 \mathrm{~cm}^{-1}\right)$ and the optical transparency of PS material is about $90 \%$. In this work, we report a strategy for realizing continuously tunable wavelength-converted wave-guiding in active nanofibers. The nanofibers were fabricated by direct drawing $\mathrm{RhB}$-doped poly(methyl methacrylate) (PMMA, refractive index $n=1.49$ ) in acetone solution. The reason for using RhB and PMMA mixed acetone solution is because PMMA exhibits extremely high optical transparency (92\%) while the evaporation of acetone is more rapidly than that of chloroform. In addition, acetone is a safe material and has been widely used in a variety of general medical and cosmetic. The experiment indicates that the self-absorption coefficient of the RhB-PMMA nanofibers is $30 \mathrm{~cm}^{-1}$ which is lower than the RhB-PS nanofiber $\left(50 \mathrm{~cm}^{-1}\right)$ while this fabrication is a facile and low cost method. Moreover, the nanofibers exhibit relatively high PL efficiency and high photobleaching resistance under an optical pump. When the pump light was evanescently coupled into the nanofibers, the excited light in the nanofibers got self-absorption and reemitted at a longer wavelength, causing a gradual wavelength conversion during propagation. On the basis of this wavelength-converted wave-guiding, nanoscale 
wavelength-converted splitters as proof-of-concept devices are demonstrated by assembling the nanofibers into crossed structures.

\section{Results}

Nanofiber fabrication and characterization. The RhB-doped polymer nanofibers were fabricated by a direct drawing method ${ }^{16}$ from polymer solutions with dissolved fluorescent dyes (see Methods). Asfabricated nanofibers have lengths up to several millimeters with diameters from 200 to $800 \mathrm{~nm}$, which is an optimal diameter for guiding visible light with respect to easy handling and efficient in/ out coupling. It should be pointed out that the lengths and diameters of the RhB-doped fibers can be adjusted by changing the viscosity of the solution and/or drawing speed. For example, the fibers with large diameter can be obtained by increasing viscosity of the solution or decreasing the drawing speed. For experimental uses, a segment was cut from the as-fabricated nanofiber, then transferred and placed on an $\mathrm{MgF}_{2}$-coated glass substrate $(n=1.50)$. Each end of the nanofiber was fixed by a microstage. The thickness of the $\mathrm{MgF}_{2}(n=1.39)$ coating is $2-5 \mu \mathrm{m}$. Figure 1a shows a scanning electron microscope (SEM) image of a coiled single nanofiber (average diameter: $\sim 480 \mathrm{~nm}$, length: $\sim 1.32 \mathrm{~mm}$ ), in which the maximum diameter variation $\Delta D \approx 50 \mathrm{~nm}$ over a length $L=1.32 \mathrm{~mm}$, and the calculated diameter variation ratio $\Delta D / L \approx 3.8 \times 10^{-5}$. Figure $1 \mathrm{~b}$ shows a typical SEM image of a $390-\mathrm{nm}$ diameter nanofiber. The close-up view of the nanofiber (inset of Fig. 1b) indicates that the nanofiber has a uniform diameter and smooth sidewall, which is favorable for low-loss optical wave-guiding. To further examine surface roughness of the nanofibers, a high-resolution SEM $(15 \mathrm{KV}, \times 100,000)$ was performed. It was found that a $280-\mathrm{nm}$ diameter nanofiber surface exhibits a root mean-square roughness of about $6 \mathrm{~nm}$ (Fig. 1c). Intensity-normalized absorption and photoluminescence (PL) spectra of RhB-doped films and nanofibers are shown in Fig. 1d. The absorption spectrum of the nanofibers shows a peak at $556 \mathrm{~nm}$ due to the absorption of the doped-RhB molecules, which was also confirmed by inset of Fig. 1d (PMMA is transparent in the visible spectral range). The PL peak of nanofibers is at $580 \mathrm{~nm}$, which is red-shift by about $5 \mathrm{~nm}$ with respect to the reference films mainly due to self-absorption.
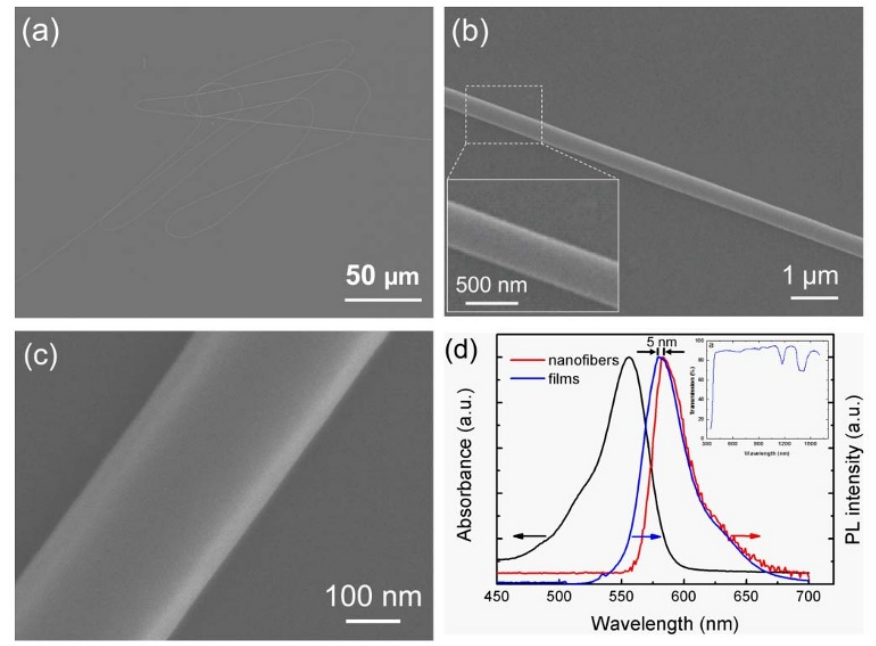

Figure 1 | Characterization of RhB-doped nanofibers. (a) SEM image of a coiled single nanofiber (average diameter: $\sim 480 \mathrm{~nm}$, length: $\sim 1.32 \mathrm{~mm}$ ). (b) SEM image of a representative 390-nm diameter straight nanofiber. Inset: close-up view of the nanofiber. (c) HR-SEM image of a 280-nm diameter nanofiber. (d) Intensity-normalized absorption and PL spectra of reference films and nanofibers. Inset: transmission spectrum of PMMA.
Optical characterization. To investigate optical properties, a single RhB-doped nanofiber (diameter: $\sim 480 \mathrm{~nm}$ ) was deposited on a $\mathrm{MgF}_{2}$-coated glass substrate. A schematic of the optical setup is shown in Fig. 2a. Evanescent coupling was used to couple the excitation light efficiently into polymer nanofiber due to its high efficiency and high compactness for broadand application ${ }^{4,19}$. A 532-nm green light (continuous-wave) using as an excitation light was evanescently coupled into the single nanofiber by a tapered fiber 1. The excited yellow light from the doped RhB was evanescently picked up using a tapered fiber 2 . The power of the excited yellow light was detected by an optical power meter (OPHIR NOVA II) integrated with a photodiode (OPHIR PD300-UV). Figure 2b shows the optical microscope image of the emitted yellow light $(\sim 580 \mathrm{~nm})$ which was excited by the injected $532-\mathrm{nm}$ green light with an optical power of $P_{\text {in }}=0.12 \mu \mathrm{W}$. The coupling length between the tapered fiber 1 and the nanofiber is about $35 \mu \mathrm{m}$. Since the tapered fiber 1 and the nanofiber is in parallel and contacted each other tightly by the van der Waals and electrostatic forces, the coupling efficiency $\eta$ reached up to $90 \%$, which can be obtained according to our reported method $^{20}$. To calculate emission efficiency of the excited yellow light from the nanofiber, we define $\xi$ $=P_{\mathrm{em}} /\left(P_{\text {in }} \times \eta\right)$, where $P_{\mathrm{em}}$ is the emitted yellow light power at the output end of the nanofiber, $P_{\text {in }}$ is the injected green light power at the input end of the nanofiber. By measuring the output yellow light power $(\sim 3.6 \mathrm{nW})$ at the end of the nanofiber (effective length of about $230 \mu \mathrm{m}$ ), an estimated emission efficiency $\xi \approx 3.7 \%$ for $\eta \sim$ 0.8 was calculated at $P_{\text {in }}=0.12 \mu \mathrm{W}$. It is worth noting that the emission efficiency of the yellow light is related to quantum efficiency of the dyes, self-absorption of the dyes, and propagation loss of the excited yellow light.

The absorption of the injected green light in the nanofiber was estimated by a cutback method (i.e., measuring the output optical power at different positions along the nanofiber) using the tapered fiber 2 as shown in Fig. 2a, which was connected to an optical power meter. Figure $2 c$ shows the measured normalized optical intensity. It was found that the intensity is exponentially decreased with increasing propagation distance along the nanofiber, obeying the LambertBeer law. The green line in Fig. $2 c$ is the fitting curve of the experimental data according to first order exponential decay fit. The estimated absorption coefficient $\alpha$ is about $80 \mathrm{~cm}^{-1}$ for $532-\mathrm{nm}$ green light, which is about one-third of that of $\mathrm{CdSe} / \mathrm{ZnS}$-doped nanofiber $\left(247 \mathrm{~cm}^{-1}\right)^{21}$. The relatively high absorption coefficient indicates that the injected green light was efficiently absorbed by the nanofibers, which benefits active nanofibers.

Self-absorption of the excited yellow light in the nanofiber was mostly caused by the overlap between absorption and PL spectra of the nanofiber. It was measured by the cutback method mentioned above. Similarly, the normalized PL intensity is also exponentially decreased with increasing propagation distance along the nanofiber as shown in Figure 2d. The yellow line is the fitting curve of the experimental data which was obtained by the Beer-Lambert law. The self-absorption coefficient $\gamma$ is about $30 \mathrm{~cm}^{-1}$ for the excited 580 -nm yellow light, which is less than as those reported in RhBPS nanofiber $\left(50 \mathrm{~cm}^{-1} \text {; RhB, } 0.05 \mathrm{wt} \%\right)^{17}$, and is one or two orders of magnitude lower than that of R6G-PMMA nanofiber (200$1200 \mathrm{~cm}^{-1}$; R6G, $\left.0.2-0.5 \mathrm{wt} \%\right)^{22}$ due to relatively low concentration of the doped dyes (RhB, $0.048 \mathrm{wt} \%$ ).

Photobleaching is one of the most important concerns for active nanofibers, which was measured by collecting PL emissions at the positions with an interval of about $5 \mathrm{~min}$ through the confocal Raman microscope and shown in Fig. 2e. One can see that the normalized PL intensity almost remains unchanged from 0 to $70 \mathrm{~min}$, but an obvious photobleaching starts after $70 \mathrm{~min}$, obeying an exponential decay. After $260 \mathrm{~min}$, about $60 \%$ of initial PL intensity still remains. It should be pointed out that since the degradation tendency of the dye is still obeying an exponential decay, therefore, the 

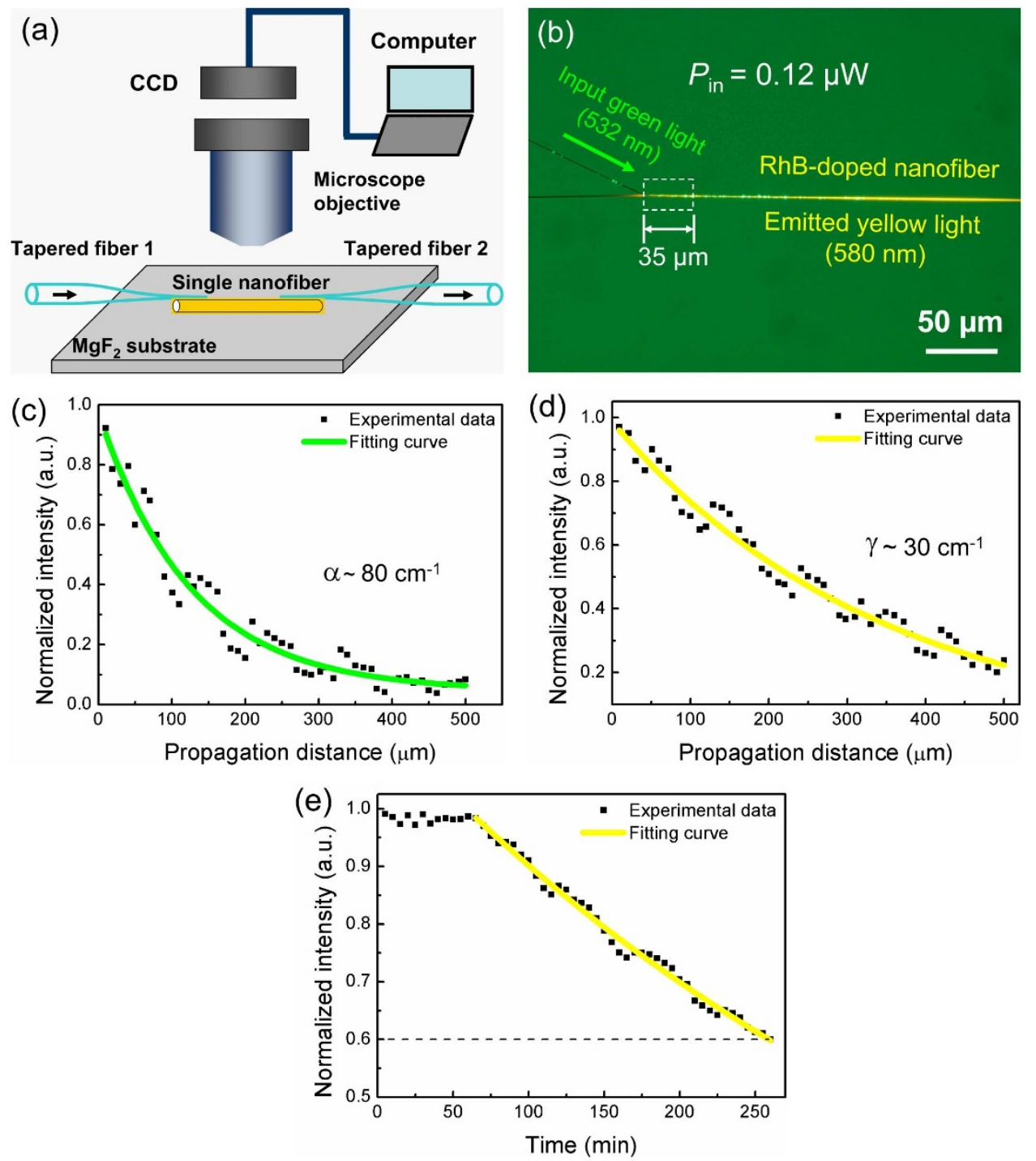

Figure 2 Optical characterization of an RhB-doped nanofiber. (a) Schematic diagram of an experimental setup. (b) Optical microscope image of the emitted yellow light $\left(580 \mathrm{~nm}\right.$ ) excited by injecting a $532-\mathrm{nm}$ green light with an optical power of $P_{\text {in }}=0.12 \mu \mathrm{W}$. The coupling length is $35 \mu \mathrm{m}$. The green arrow shows the propagation direction of the input light. (c) Normalized optical intensity of green light in the nanofiber as a function of propagation distance. (d) Normalized PL intensity of the excited yellow light in the nanofiber as a function of propagation distance. (e) Normalized PL intensity as a function of time.

measurement was stopped after $260 \mathrm{~min}$. This is comparable with the RhB-PS nanofiber $(\sim 56 \%)^{17}$, lower than the quantum-dotdecorated nanofibers $(\sim 88 \%)^{23}$, and higher than the irradiated dye-doped nanofibers $(\sim 25 \%)^{24}$. Furthermore, the resistance to photobleaching can be further improved by lowering concentration of the doped dyes and excitation power. This makes the nanofibers a promising candidate for practical applications due to their high photobleaching resistance.

Device assembly and characterization. By utilizing the self-absorption and re-emission effect of the excited light in dye-doped nanofibers, wavelength-converted wave-guiding with high PL efficiency can be realized. In the experiment, the PL spectra were measured by an USB $2000+$ fiber optic spectrometer (Ocean Optics Inc., USA) from the tapered fiber 2. For example, by launching the $532-\mathrm{nm}$ green light $(0.12 \mu \mathrm{W})$ into a 480 -nm diameter nanofiber, the nanofiber exhibited different emission colors such as yellow-orange, orange, and redorange at the positions of $A_{1}, A_{3}$, and $A_{3}$, respectively (Fig. 3a). The corresponding PL peaks (i.e., maximum PL wavelength, also referred to as center wavelength $\lambda_{c}$ ) for the positions $A_{1}, A_{3}$, and $A_{3}$ are 583,592 , and $597 \mathrm{~nm}$, respectively. Moreover, the PL intensity at the positions of from $A_{1}$ to $A_{3}$ became weaker and weaker due to self-absorption of excited light (Fig. 3b). When the propagation distance was increased to about $150 \mu \mathrm{m}$ (from $\mathrm{A}_{1}$ to $\mathrm{A}_{2}$ ), the guided yelloworange light $(583 \mathrm{~nm})$ in the nanofiber got self-absorption and reemitted at a longer wavelength (orange, $592 \mathrm{~nm}$ ) causing a 9-nm PL peak variation in the emitted PL spectrum. When the propagation distance was changed to about $100 \mu \mathrm{m}$ (from $\mathrm{A}_{2}$ to $\mathrm{A}_{3}$ ), the emission color at the position $\mathrm{A}_{3}$ was changed to red-orange $(597 \mathrm{~nm})$, resulting a $5 \mathrm{~nm} \mathrm{PL}$ peak variation. The estimated emission efficiency at the output end of the nanofiber is about $1.5 \%$. The demonstration shows that the PL peak variation of the guided PL spectrum in the nanofiber did not increase linearly with propagation distance due to nonlinear self-absorption of the excited yellow, which generally experienced a first rapid increase, followed by a slow increase and finally got saturated with an increase in propagation distance (see Supplementary Fig. S1). It is worth mentioning that the emission color continuously changes from yellow-orange to red-orange in the nanofiber was not caused by the short-pass filtering effect ${ }^{25}$, which demonstrates higher 

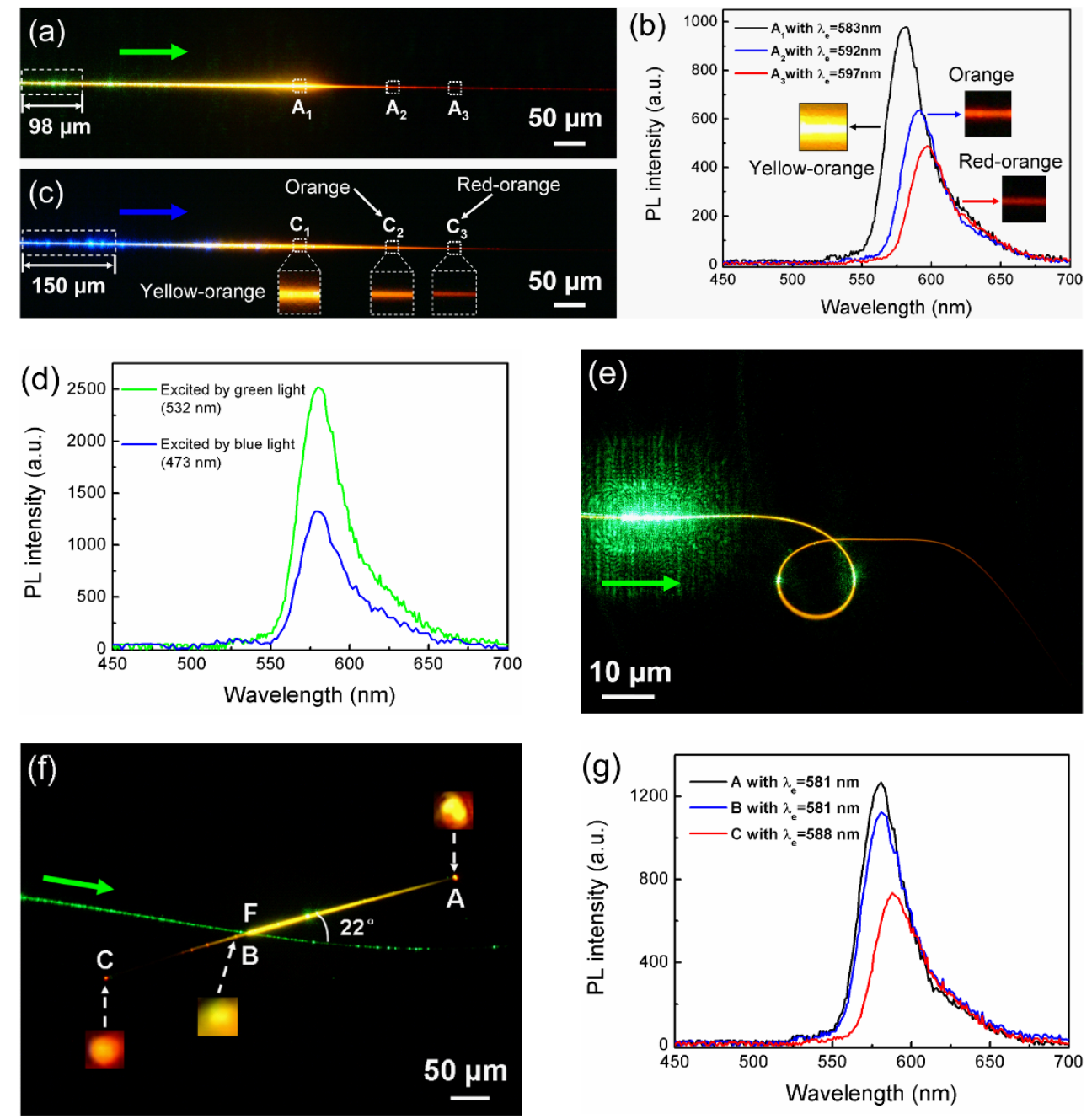

Figure 3 Optical microscope images and PL spectra of nanofibers. A 480-nm diameter nanofiber excited by $0.12 \mu \mathrm{W}$ (a, b) $532-\mathrm{nm}$ and (c) 473 -nm light. (d) PL spectra of the nanofiber under excitation lights of 473-nm and 532-nm wavelengths at power of $0.12 \mu \mathrm{W}$. (e) Optical microscope image of a nanofiber with average diameter of about $300 \mathrm{~nm}$ excited by 532-nm green light with $P_{\text {in }}=0.12 \mu \mathrm{W}$. (f) Optical microscope image and (g) PL spectra at positions A, B, and C of a 480-nm diameter nanofiber excited by 532-nm green light with $P_{\text {in }}=0.12 \mu \mathrm{W}$ from a tapered fiber. The arrows show the propagation direction of the input light.

radiation losses for longer wavelengths for a specific nanofiber (i.e., the longer wavelength light such as red-orange light would propagate shorter distance than that of yellow light along the nanofiber). On the other hand, it was also not due to the superposition of the injected green light and the excited yellow light in the nanofiber because only intermediate colors between green and yellow rather than yelloworange, orange, or red-orange can be obtained according to color mixing theory ${ }^{26}$. A Similar phenomenon was also observed by launching the $473-\mathrm{nm}$ blue light $(0.12 \mu \mathrm{W})$ into it. Figure $3 \mathrm{c}$ shows that the nanofiber exhibited different emission colors such as yelloworange $(582 \mathrm{~nm})$, orange $(590 \mathrm{~nm})$, and red-orange $(595 \mathrm{~nm})$ at the positions $C_{1}, C_{2}$, and $C_{3}$, respectively (see Supplementary Fig. S2). The resulting PL peak variation of the guided PL spectrum was decreased from $8 \mathrm{~nm}$ to $5 \mathrm{~nm}$ when the propagation distance was decreased from $150 \mu \mathrm{m}$ (from $\mathrm{C}_{1}$ to $\mathrm{C}_{2}$ ) to $100 \mu \mathrm{m}$ (from $\mathrm{C}_{2}$ to $\mathrm{C}_{3}$ ). The estimated emission efficiency at the output end of the nanofiber was about $1.0 \%$. By comparing Fig. 3a with Fig. $3 c$, one can see that the PL intensity excited by green light is stronger than that of excited by blue light. This was confirmed by Fig. $3 \mathrm{~d}$. The emission efficiency excited by the $532-\mathrm{nm}$ green light is about 1.8 times higher than that of excited by the 473-nm blue light (Fig. $3 \mathrm{~d}$ ).

To investigate the propagating light mode of this nanofiber, we suspended a single nanofiber with average diameter of about $300 \mathrm{~nm}$ in air, the $532-\mathrm{nm}$ green light $(0.12 \mu \mathrm{W})$ was evanescently coupled into it using a tapered fiber (Fig. 3e). The green light appeared in the ring constructed with the nanofiber is due to the illumination of light leaked out from the neared tapered fiber. From Fig. 3e, it can be seen that yellow light was generated along the nanofiber but no waveguiding was observed at the output end of the fiber. This is due to that the diameter of the nanofiber is gradually decreased and optical loss is increased. Light in the nanofiber propagates in the transverse magnetic modes (TM modes) and the numbers of possible modes, $m$, can be simply given by $^{27}$

$$
m<(2 d / \lambda)\left(n_{\mathrm{nw}}^{2}-n_{0}^{2}\right)^{0.5}
$$

where $\lambda=580 \mathrm{~nm}$ is the wavelength of the propagating light, $n_{\mathrm{nw}}=$ 1.49 and $n_{0}=1$ are the refractive indices of the nanofiber and air, respectively, and $d(\sim 300 \mathrm{~nm})$ is the nanofiber diameter. According to Eq. (1), the number of the propagation mode was calculated as $m<1.2$, suggesting only one allowable mode for the light propagation within the nanofiber. To transmit single-mode light $(580 \mathrm{~nm})$, required minimum diameter for the nanofiber is $d_{\min }=262 \mathrm{~nm}$. Because the fiber diameter of the output end is decreased to less than $262 \mathrm{~nm}$, so there is no wave-guiding was observed.

It would be desirable to operate with different light emissions for integrated photonic circuits, therefore, we systematically investigated 
a crossed structure that generates several colors of light and separates two of them in two opposite directions in a nanofiber. Figure $3 \mathrm{f}$ shows that the $532-\mathrm{nm}$ green light $(0.12 \mu \mathrm{W})$ was again evanescently coupled into a $480-\mathrm{nm}$ diameter nanofiber by a tapered fiber through a crossed structure, and the output optical signals from its both ends are detected simultaneously using two tapered fibers. Since the coupling efficiency decreases with increasing cross angle between the tapered fiber and the nanofiber for a specific wavelength and sturcture $^{20}$, therefore, most of the launched green light was coupled into the right part of the nanofiber which forms a cross angle of $22^{\circ}$ with the tapered fiber, however, almost none green light was coupled into the left part of the nanofiber from the tapered fiber. Here we defined as coupling efficiency $\eta=P_{\text {out }} / P_{\text {ex }}$, where $P_{\text {ex }}$ is the launched 532-nm green light power $(0.12 \mu \mathrm{W})$ and $P_{\text {out }}$ is the measured output $532-\mathrm{nm}$ green light power $(\sim 19 \mathrm{nW})$ at the end of the right part of the nanofiber, and the estimated $\eta$ is about $16 \%$, which was estimated by using a same diameter pure PMMA nanofiber instead of the RhBdoped PMMA nanofiber. When the propagation distance was increased to about $270 \mu \mathrm{m}$ (from F to A), the excited yellow light $(580 \mathrm{~nm})$ at the junction $\mathrm{F}$ was shifted to a longer wavelength light (yellow, $581 \mathrm{~nm}$ ) at the position of A (Fig. 3g). The bright spot observed at the position A is due to out-coupling of PL caused by diffraction. A fraction of yellow light at the position A was coupled back to the nanofiber and transmitted along the nanofiber almost without self-absorption before reaching the junction $\mathrm{F}$. This also was confirmed by the scattered yellow-orange $(582 \mathrm{~nm})$ spot at the position $\mathrm{B}$ which close to the junction $\mathrm{F}$ (the distance between the positions $\mathrm{F}$ and $\mathrm{B}$ is $d_{\mathrm{FB}}=20 \mu \mathrm{m}$ ). The yellow-orange light at position $\mathrm{B}$ was sequentially propagates in the nanofiber and again got selfabsorption and reemitted at $588 \mathrm{~nm}$ (orange) at the position C. Above demonstration indicates that wavelength-converted waveguiding can be realized through a crossed structure. Moreover, the desirable wavelength can be tuned by changing the propagation distance.

To further demonstrate multi-channel wavelength-converted wave-guiding ability of the nanofiber, a $2 \times 2$ splitter with a branching angle of $20^{\circ}$ was formed by twisting two nanofibers with diameters of $553 \mathrm{~nm}$ (branch A-2) and $475 \mathrm{~nm}$ (branch B-1) (Fig. 4a). The coupling region is about $12 \mu \mathrm{m}$ long and $1.0 \mu \mathrm{m}$ wide. By launching the $532-\mathrm{nm}$ green light $(0.12 \mu \mathrm{W})$ into the branch A via evanescent coupling, the excited yellow light $(580 \mathrm{~nm})$ was propagated over a distance of about $98 \mu \mathrm{m}$ and divided into the output branches 1 and 2 with a splitting ratio of about 54:46, finally, the colors of the guided light in the branches 1 and 2 were both changed from yellow $(580 \mathrm{~nm})$ to yellow-orange $(581 \mathrm{~nm})$ over a distance of about $190 \mu \mathrm{m}$ (Fig. 4b). The guided yellow-orange light at the end of the branch 2 was coupled back to the branch $B$, and then was changed to orange $(587 \mathrm{~nm})$ over a distance of about $112 \mu \mathrm{m}$. It should be pointed out that in our optical propagation experiment, the optical losses can be neglected because the self-absorption coefficient ( $30 \mathrm{~cm}^{-1}$, corresponding to a loss coefficient of about $13 \mathrm{~dB} / \mathrm{mm}$ ) is higher than the optical losses $(0.01 \sim 0.1 \mathrm{~dB} / \mathrm{mm})$ for the excited 580 -nm yellow light. Additionally, the branching angle also affect device performance, the dependence of the branching angle on the output power at the branches was also investigated (see Supplementary Fig. S3). Similarly, a $3 \times 3$ splitter was also formed by twisting three nanofibers with diameters of 610,508 , and $545 \mathrm{~nm}$ for branches $\mathrm{A}$ to $\mathrm{C}$ (Fig. $4 \mathrm{c})$. The 532 -m green light $(0.12 \mu \mathrm{W})$ was launched into the branch $\mathrm{A}$, the excited yellow light $(580 \mathrm{~nm})$ was transmitted over about $70 \mu \mathrm{m}$ and divided into the branches 1,2 , and 3 with a splitting ratio of about $38: 31: 31$, finally, the guided yellow light $(580 \mathrm{~nm})$ was changed to yellow-orange $(582 \mathrm{~nm})$, yelloworange light $(581 \mathrm{~nm})$, and yellow-orange light $(581 \mathrm{~nm})$ at the branches 1,2, and 3, respectively. The output light for the branches $\mathrm{B}$ and $\mathrm{C}$ was changed from yellow-orange $(582 \mathrm{~nm})$ to orange $(587 \mathrm{~nm})$ with the power ratio about $50: 50$. By comparing Fig. $4 \mathrm{~b}$
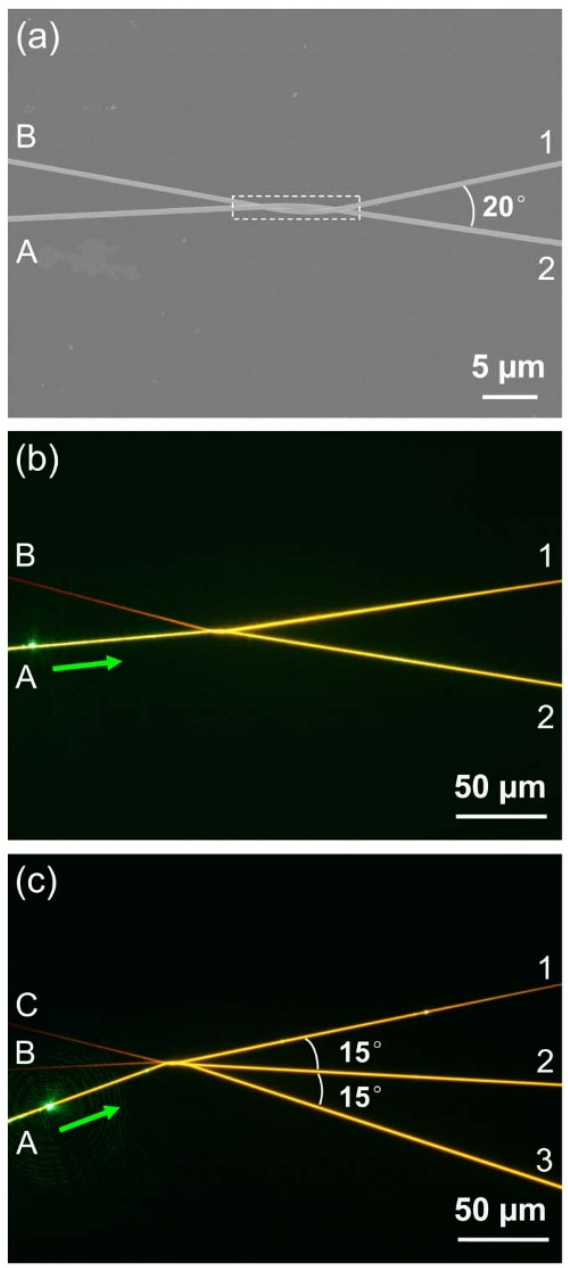

Figure $4 \mid 2 \times 2$ and $3 \times 3$ splitters. (a) SEM image of a $2 \times 2$ splitter with diameters of 553 and $475 \mathrm{~nm}$ for branches A-2 and B-1, respectively. The branching angle between the branches 1 and 2 is $20^{\circ}$. (b) Optical microscope image of the same splitter as (a) by launching 532-nm green light $(0.12 \mu \mathrm{W})$ to the branch A by evanescent coupling. The green arrow shows the propagation direction of the input light. (c) A $3 \times 3$ splitter with diameters of 610,508 and $545 \mathrm{~nm}$ for branches A, B, and C, respectively. The green arrow shows the propagation direction of the input light.

and Fig. 4c, it was found that more different colors of light emission were output by increasing output ports. Above demonstrations indicate that multi-channel wavelength-converted splitters, which are of great importance for nanophotonic integration, can be performed by optimizing structure parameters such as propagation distance and branch angles.

\section{Discussion}

In this work, we demonstrate a facile strategy based on self-absorption and re-emission effect in dye-doped nanofibers for realizing continuously tunable wavelength-converted wave-guiding. The nanofibers with diameters of 200-800 nm exhibit high PL efficiency, high photobleaching resistance, relatively high absorption coefficient $\left(80 \mathrm{~cm}^{-1}\right)$ and low self-absorption coefficient $\left(30 \mathrm{~cm}^{-1}\right)$ under optical wave-guiding excitation. When the pump light was launched into the nanofibers, the excited light in the nanofibers undergoes a process of self-absorption and reemission, resulting in a gradual wavelength conversion during propagation. On the basis of this wavelength-converted wave-guiding, we have assembled the nanofibers into crossed structures, in which the functions such as wavelength-converted and output power-controlled wave-guiding have 
been performed by optimizing structure parameters such as propagation distance and branch angles. We believe that the nanofibers could serve as important optical information components in nanoscale integrated photonic systems.

\section{Methods}

Fabrication of dye-doped polymer nanofibers. The dye-doped polymer nanofibers were fabricated as follows: first, $6.2 \mathrm{~g}$ of PMMA and $3 \mathrm{mg}$ of RhB were dissolved into $20 \mathrm{~g}$ of acetone, and then the mixture was stirred at room temperature for 1 hour and followed by 30-minutes ultrasound, finally a uniform solution with an appropriate viscosity was obtained for drawing. Second, the tip of a taped silica fiber (radius: $\sim 125 \mu \mathrm{m}$ ) was immersed into the mixture solution for $1 \sim 2 \mathrm{~s}$ and then pulled out with a speed of $0.1 \sim 1 \mathrm{~m} / \mathrm{s}$, leaving a RhB-doped PMMA wire extending between the solution and the fiber tip. With very fast evaporation of the acetone and finally, a naked nanofiber doped with $\mathrm{RhB}$ was formed.

Light sources. The monochromatic light sources (473 and $532 \mathrm{~nm}$ wavelength) used for dye-doped nanofibers excitation and characterization are continuous-wave lasers.

Optical characterization of the dye-doped nanofibers. The PL emissions of the nanofibers were collected using long working distance microscope objectives and directed to an optical power meter (OPHIR NOVA II) integrated with a photodiode (OPHIR PD300-UV) or an USB 2000+ fiber optic spectrometer (Ocean Optics Inc., USA), and a CCD camera (DXM 1200F, Nikon), respectively. The optical absorption and photobleaching properties of the nanofibers were measured by collecting PL emissions through the confocal Raman microscope (WITec Ltd., alpha300 S).

1. Psaltis, D. Coherent optical information systems. Science 298, 1359-1363 (2002).

2. Foster, M. A. et al. Broad-band optical parametric gain on a silicon photonic chip. Nature 441, 960-963 (2006)

3. Sirbuly, D. J. et al. Optical routing and sensing with nanowire assemblies. Proc. Natl. Acad. Sci. U. S. A. 102, 7800-7805 (2005).

4. Xing, X., Zhu, H., Wang, Y. \& Li, B. Ultracompact photonic coupling splitters twisted by PTT nanowires. Nano Lett. 8, 2839-2843 (2008).

5. Zhu, Y., Hu, X., Lu, C., Huang, Y. \& Gong, Q. All-optical tunable wavelengthdivision multiplexing based on colloidal crystal coated silver film. Plasmonics 7, 589-594 (2012).

6. Matsuura, M., Raz, O., Calabretta, N. \& Dorren, H. J. S. Multichannel wavelength conversion of 50-Gbit/s NRZ-DQPSK signals using a quantum-dot semiconductor optical amplifier. Opt. Express 19, B560-B566 (2011).

7. Chiu, H. K., Chang, C. H., Hou, C. H., Chen, C. C. \& Lee, C. C. Wavelength selective filter based on a hollow optical waveguide. Appl. Opt. 50, 227-230 (2011).

8. Bliss, C. L., McMullin, J. N. \& Backhouse, C. J. Integrated wavelength-selective optical waveguides for microfluidic-based laser-induced fluorescence detection. Lab on chip 8, 143-151 (2008).

9. Bernard, J. E., Alcock, A. J. \& Lokhnygin, V. D. Grating-tuned, singlelongitudinal-mode, diode-pumped $\mathrm{Nd}^{\mathrm{Y}} \mathrm{YVO}_{4}$ laser. Opt. Lett. 18, 2020-2022 (1993).

10. Dai, D. \& He, S. Compact silicon-based wavelength-selective photonic integrated devices and the applications. Proc. of SPIE 7220, 72200D (2009).

11. Park, H. G. et al. A Wavelength-selective photonic-crystal waveguide coupled to a nanowire light source. Nature Photon. 2, 622-626 (2008).

12. Chen, Z. et al. Wavelength-selective directional coupling with dielectric-loaded plasmonic waveguides. Opt. Lett. 34, 310-312 (2009).
13. Xu, J. et al. Wavelength-converted/selective waveguiding based on compositiongraded semiconductor nanowires. Nano Lett. 12, 5003-5007 (2012).

14. Skirtach, A. G. et al. Nanorods as wavelength-selective absorption centers in the visible and near-infrared regions of the electromagnetic spectrum. Adv. Mater. 20, 506-510 (2008)

15. Ma, H., Jen, A. K.-Y. \& Dalton, L. R. Polymer-based optical waveguides: materials, processing, and devices. Adv. Mater. 14, 1339-1365 (2002).

16. Xing, X., Wang, Y. \& Li, B. Nanofiber drawing and nanodevice assembly in poly(trimethylene terephthalate) Opt. Express 16, 10815-10822 (2008).

17. Gu, F., Yu, H., Wang, P., Yang, Z. \& Tong, L. Light-emitting polymer single nanofibers via waveguiding excitation. ACS Nano 4, 5332-5338 (2010).

18. Song, H., Liu, L. \& Xu, L. Lasing action in dye doped polymer nanofiber knot resonator. J. Lightwave Technol. 27, 4374-4376 (2009).

19. Yu, H., Liao, D., Johnston, M. B. \& Li, B. All-optical full-color displays using polymer nanofibers. ACS Nano 5, 2020-2025 (2011).

20. He, W., Li, B. \& Pun, E. Y.-B. Wavelength, cross-angle, and core-diameter dependence of coupling efficiency in nanowire evanescent wave coupling. Opt. Lett. 34, 1597-1599 (2009).

21. Zhang, R., Yu, H. \& Li, B. Active nanowaveguides in polymer doped with CdSe/ ZnS core/shell quantum dots. Nanoscale 4, 5856-5859 (2012).

22. Camposeo, A. et al. Laser emission from electrospun polymer nanofibers. Small 5, 562-566 (2009)

23. Yu, H., Zhang, H. \& Li, B. Optical properties of quantum-dot-decorated polymer nanofibers. Nanotechnology 22, 335202 (2011).

24. Camposeo, A., Benedetto, F. D., Stabile, R., Cingolani, R. \& Pisignano, D. Electrospun dye-doped polymer nanofibers emitting in the near infrared. Appl. Phys. Lett. 90, 143115 (2007).

25. Law, M. et al. Nanoribbon waveguides for subwavelength photonics integration. Science 305, 1269-1273 (2004).

26. Schubert, E. F. Light Emitting Diodes (Cambridge Univ. Press, Cambridge, 2003).

27. Takazawa, K., Kitahama, Y., Kimura, Y. \& Kido, G. Optical waveguide selfassembled from organic dye molecules in solution. Nano Lett. 5, 1293-1926 (2005).

\section{Acknowledgments}

The authors thank S. Liang for assistance in preliminary experiment, R. Zhang for assistance in sample preparation. This work was supported by the National Natural Science Foundation of China (Grants 11274395, 10974261, and 60625404)

\section{Author contributions}

B.L. supervised the project; H.Y. performed the experiments; H.Y. and B.L. discussed the results and wrote the manuscript.

\section{Additional information}

Supplementary information accompanies this paper at http://www.nature.com/ scientificreports

Competing financial interests: The authors declare no competing financial interests.

License: This work is licensed under a Creative Commons

Attribution-NonCommercial-ShareALike 3.0 Unported License. To view a copy of this license, visit http://creativecommons.org/licenses/by-nc-sa/3.0/

How to cite this article: Yu, H.Q. \& Li, B.J. Wavelength-converted wave-guiding in dye-doped polymer nanofibers. Sci. Rep. 3, 1674; DOI:10.1038/srep01674 (2013). 